\title{
Scanning probe oxidation of SiC, fabrication possibilities and kinetics considerations
}

\author{
M. Lorenzoni ${ }^{\mathrm{a})}$ and B. Torre \\ Nanophysics, Istituto Italiano di Tecnologia, via Morego, 30, 16163 Genova, Italy
}

(Received 23 July 2013; accepted 28 September 2013; published online 16 October 2013)

\begin{abstract}
We report the outcome of atomic force microscopy local anodic oxidation experiments on $6 \mathrm{H}-\mathrm{SiC}$ in air. Oxide thickness can be easily tuned by varying applied voltage and pulse duration. The height and the aspect ratio of single dots produced by single DC pulses are remarkably higher than what was reported previously, with self limiting heights exceeding $100 \mathrm{~nm}$. We propose that the diminished density and the change in chemical composition of the oxide grown on $\mathrm{SiC}$ with respect to oxide grown under similar condition on Si cause a drop in the activation energy of oxanions diffusion within the newly formed oxide layer. (C) 2013 AIP Publishing LLC.

[http://dx.doi.org/10.1063/1.4825265]
\end{abstract}

Silicon carbide is a wide gap semiconductor extensively employed in microelectronics industry. Due to its high thermal conductivity, high temperature operation, and chemical inertness, it is used as a valuable alternative to silicon for devices operating in hard conditions. Remarkably, $\mathrm{SiC}$ is the only semiconductor (except $\mathrm{Si}$ ) whose native oxide is $\mathrm{SiO}_{2}$. However, $\mathrm{SiC}$ oxidation could result in mixed oxides products containing $\mathrm{C}$ inclusions. State-of-the-art technology in patterning semiconductor substrates mainly relies on mask-based techniques such as optical lithography or mask-less techniques like electron beam lithography, which are particularly suited for industrial applications such as large scale production in microelectronics and microfabrication, in general. Among all the alternatives, several promising scanning probe-related lithographies (SPLs) also emerged $^{1-4}$ as an affordable and very versatile nanofabrication technique. The advantages of using an atomic force microscope (AFM) reside in the in-line nanometric accuracy and in the possibility of directly applying multistep processes on pre-patterned substrates with no need for photoresist coatings and/or alignment tools. This makes SPL an ideal tool for flexible and fast prototyping of custom nanodevices. Few works have addressed the tip induced oxidation of $\mathrm{SiC}$ (Refs. 5-7) and none extensively explored the kinetics involved.

In this work, we present the outcome of field induced oxidation (FIO) experiments in contact mode on $6 \mathrm{H}-\mathrm{SiC}$ (0001) surface including: single pulse oxidation (pulse time ranging from 0.1 to $30 \mathrm{~s}$ ), patterning examples (single lines and 3D patterns), and HF etch tests. The schematic of FIO is depicted in Fig. 1(a). By means of preliminary HF etching, we removed $\mathrm{SiC}$ native oxide layer to end up with an hydroxyl terminated surface. ${ }^{8}$ On such surface, we were able to produce controlled single oxide dots for long oxidation times ( $>10 \mathrm{~s})$, obtaining unexpected height and aspect ratio (height $\approx 100 \mathrm{~nm}$, full width at half maximum $(\mathrm{FWHM}) \approx 350 \mathrm{~nm})$ in comparison with most FIO experiments (Figs. 1(b) and 1(c)). In order to have a quantitative explanation of the phenomenon, we compared the experimental data with available models dealing with $\mathrm{Si}$ probe

${ }^{\text {a)} E l e c t r o n i c ~ m a i l: ~ m a t t e o . l o r e n z o n i @ i i t . i t ~}$ oxidation in air. We estimated both oxide density and expansion rate by means of a wet etching test (dilute aqueous HF dissolves selectively $\mathrm{SiO}_{2}$ ). With respect to $\mathrm{SiO}_{2} / \mathrm{Si}$ case, a diminished density indicates also possible carbon incorporation within the oxide, leading to a $\mathrm{SiO}_{x} \mathrm{C}_{y}$ stoichiometry. Finally, we verified the presence of residual trapped charges by measuring the surface potential by Kelvin probe force microscopy (KPFM) of freshly fabricated oxide patterns. Both measurements suggest the presence of a residual conductance in the oxide that is consistent with the remarkably higher thickness of the fabricated oxide layer. In order to assess the insulating nature of oxide features on $\mathrm{SiC}$, we also performed conductive AFM measurements. ${ }^{9}$

Experiments analyzed were performed over the range of $20 \%-45 \%$ RH (accuracy of 1\%). In all experiments, the tip was biased negatively in respect to the sample (sample positive), in the text the voltage values reported are sample voltages. Kelvin probe force microscopy measurements have been performed by means of an Asylum MFP 3D in dry nitrogen flux $(\mathrm{RH}<4 \%)$ at room temperature using Pt coated probes. The work function of the tip $\left(\Phi_{\text {tip }}=4.93 \pm 0.05 \mathrm{eV}\right)$ was carefully calibrated by KPFM on freshly cleaved highly oriented pyrolytic graphite (HOPG). ${ }^{10}$

In Fig. 2, we present two examples of fabricated patterns by SPL on SiC. Figure 2(a) shows the topography and the height profile in case of single lines written at $1 \mu \mathrm{m} \mathrm{s}^{-1}$ as a function of bias; lines are uniform, between $5 \mathrm{~V}$ and $13 \mathrm{~V}$ both height, and FWHM increase linearly with the applied value of bias, similarly to what happens during FIO on $\mathrm{Si}$, between $13 \mathrm{~V}$ and 15 lines become wider but height drops to $\approx 3 \mathrm{~nm}$. The minimum lateral feature size obtained is $\approx 50 \mathrm{~nm}$, compatible with resolution limits of contact mode SPL. ${ }^{11}$ Figure 2(b) shows three different patterns fabricated during a single scan at different voltage values, demonstrating the single pass control capability on the $3 \mathrm{D}$ features produced. As shown in the height profile of Fig. 2(b), features height can be finely tuned with $0.4 \mathrm{~nm}$ accuracy allowing to fabricate $3 \pm 0.4 \mathrm{~nm}$ (left gear) and $5 \pm 0.4 \mathrm{~nm}$ (right gear) extended features within the same image. It is worth to notice that thick oxide masks obtained on $\mathrm{SiC}$ could be effectively used during inductively coupled plasma reactive ion etching 
(a)
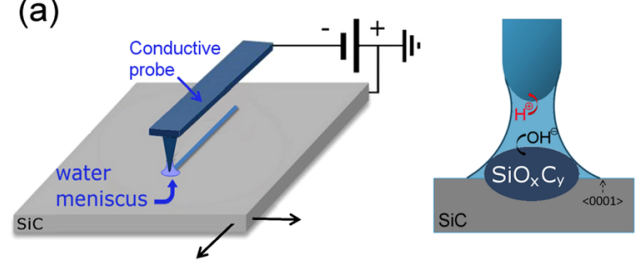

(b)

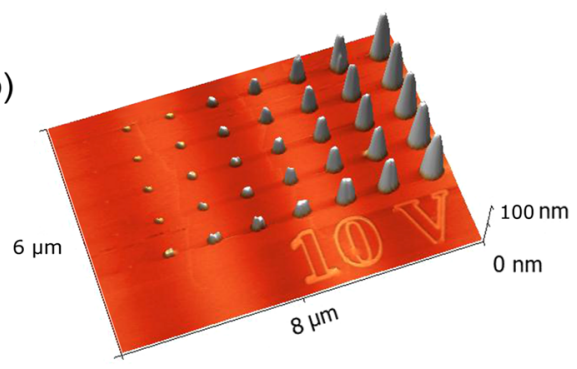

(c)
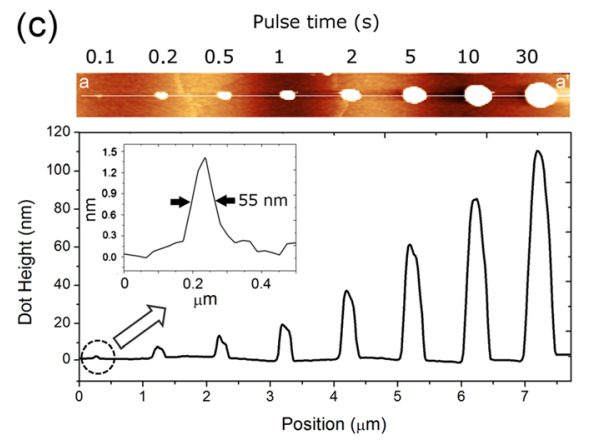

FIG. 1. In (a), schematic diagram of local anodic oxidation. (b) AFM 3D topography of an array of single dots produced by field induced oxidation in contact mode on $6 \mathrm{H}-\mathrm{SiC}$ surface with a relative humidity of $40 \%$. Each line is obtained by the same pulse time variation, eight pulses of $10 \mathrm{~V}$ ranging from 0.1 to $30 \mathrm{~s}$. In (c), a detail of the topography with the height profile. The inset in (b) is given to better visualize the profile of the dot obtained by a $0.1 \mathrm{~s}$ pulse.

of silicon carbide, obtaining an even more effective fabrication technique, with respect to the case of $\mathrm{SiO}_{2} / \mathrm{Si} \mathrm{SPL}$ grown masks. ${ }^{4}$ Even if $\mathrm{SiC}$ behavior in fluorinated plasmas is different from $\mathrm{Si}$ (with etch rate ratios never exceeding unity), satisfactory etch rates have been obtained on $6 \mathrm{H}-\mathrm{SiC}$ using chloride-based plasmas with $\mathrm{SiO}_{2}$ masks. ${ }^{12}$ Figure 3 illustrates a fabricated pattern consisting of multiple $1 \times 1 \mu \mathrm{m}$ oxide squares formed by parallel lines written at $1 \mu \mathrm{m} \mathrm{s}{ }^{-1}$ and $10 \mathrm{~V}$ bias, before (Fig. 3(a)) and after (Fig. 3(b)) wet etching (HF, $5 \mathrm{wt} . \%$ for $30 \mathrm{~s}$ ). The squared patterned imaged by AFM has been readily wet etched and the resulting AFM topography of the original and etched pattern is detailed in Fig. 3(c). The molar volume mismatch between $\mathrm{Si}$ and its native oxide has been widely investigated; something very similar happens during SiC FIO. Expansion factor $\nu^{*}$ is defined as the ratio between oxide and substrate molar volumes: in case of unidirectional expansion, it can be alternatively evaluated from height measurements, in fact, if the expansion is constrained in one direction $\nu^{*}$ is equal to $(h+d) / d$, where $h$ is the measured oxide height and $d$ is the HF etch depth (see Fig. 3(c)). In our case, geometrical parameters have been carefully evaluated from spatial average on extended ( $1 \mu \mathrm{m} \times 1 \mu \mathrm{m}$ square) features before and after etching, which also allows to avoid tip convolution artifacts at the step edges and related underestimation of $d$ in case of narrow features and finite tip half cone. ${ }^{13}$ For $\mathrm{SiO}_{2} / \mathrm{Si} \mathrm{SPL}$

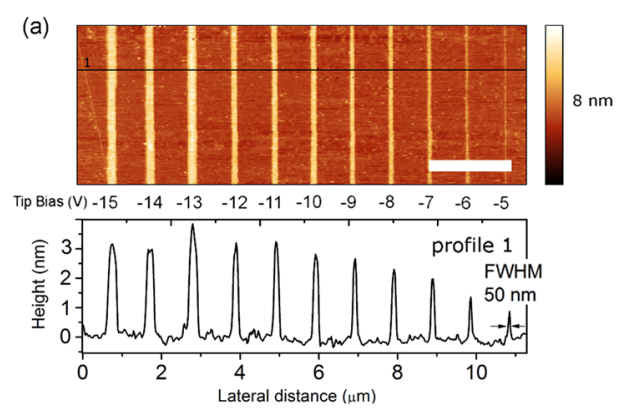

(b)
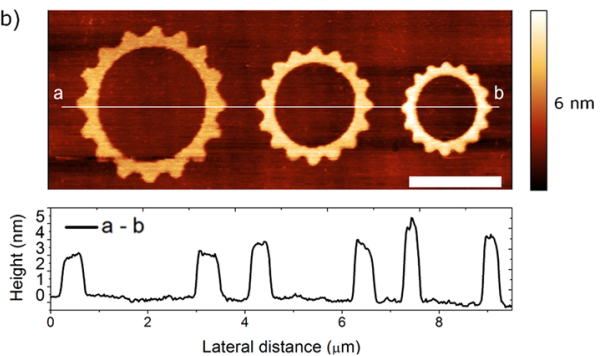

FIG. 2. Examples of oxide patterns. In (a), AFM topography and profile of single continuous lines written at $1 \mu \mathrm{m} \mathrm{s}^{-1}$ and different tip bias applied. As expected, oxide height and width increases with bias. In (b), pattern fabricated during a single scan, a grayscale bitmap image was converted into a map of voltage values used during the scan thus obtaining a $3 \mathrm{D}$ control on the features produced. The control on patterned oxide height is achieved by tuning the voltage while the tip is scanning in contact mode. From left to right, the three gears were produced by 12,15 , and $20 \mathrm{~V}$, respectively. Scale bar is $2 \mu \mathrm{m}$ in both images.

oxides, a value of $\nu_{S i}{ }^{*} \approx 2.65$ is reported, ${ }^{14}$ while in the case of $\mathrm{SiC}$ substrate, we measured $\nu_{S i C}{ }^{*}=2.49$ (Fig. 3 and Table I). The density of SPL oxide grown on $\mathrm{Si}$ is known to be lower than the density of thermal oxide, ${ }^{15}$ as a reference we can consider that the density of a thermal-wet grown film is $\approx 2.2 \mathrm{~g} \mathrm{~cm}^{-3}$. Using a molar mass $\mathrm{M}_{\mathrm{SiO} 2}$ of $60.08 \mathrm{~g} \mathrm{~mol}^{-1}$, we obtain an estimation of the density $\left(\rho=M / m^{*}\right)$ equal to 1.93 $\mathrm{g} \mathrm{cm}^{-3}$ for SPL oxide grown on $\mathrm{SiC}$ as expected for SPM oxides. Interestingly, as more carbon is incorporated into $\mathrm{SiO}_{2}$ films grown on $\mathrm{Si}$ by chemical vapor deposition (CVD), both the film density and refractive index decreases. ${ }^{16}$ This could indicate a similar carbon inclusion during SPL oxidation of SiC. In Table I, we made a comparison between the experimental expansion factors and the calculated values for different possible composition of the oxide obtained on $\mathrm{SiC}$; the possible reactions are taken into consideration as shown in the lower part of Table I, with carbon content in the product of oxidation ranging from $0 \%$ (pure $\mathrm{SiO}_{2}$ ) to almost $20 \%$ (silicon oxycarbide with high $\mathrm{C}$ content). The experimental outcome $\left(2.49 \mathrm{~g} \mathrm{~cm}^{-3}\right)$ agrees well with a low content in carbon that presents a density $\approx 2.32 \mathrm{~g} \mathrm{~cm}^{-3}$.

The mechanism and kinetics of Si oxidation surfaces by AFM has been extensively studied, ${ }^{17-20}$ since the very first experiments. The use of low temperature oxidation theory ${ }^{21}$ alone was unable to account for experimental evidence, therefore, several other theories were raised to explain different regimes. In our case, oxide thickness shows a linear dependence on the applied bias and a direct logarithmic dependence on the voltage pulse duration, which remarkably differs from conventional electrochemical anodization that is well described by an inverse log-of-time analytical function. This deviation from conventional growth kinetics is explained by the cooperative effect of water content into the system 
(a)

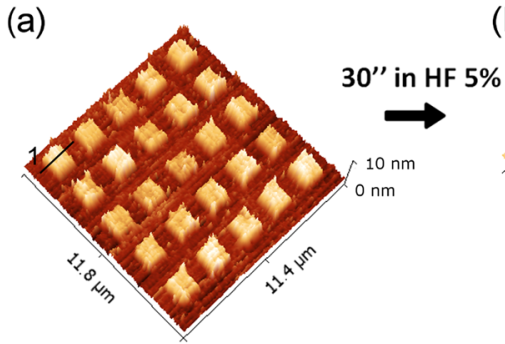

(b)

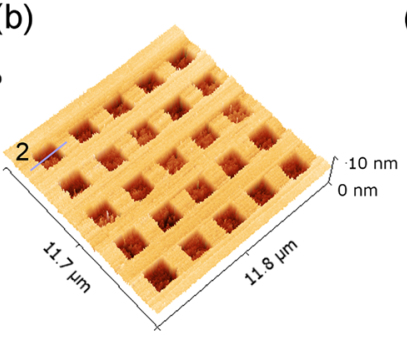

(c)

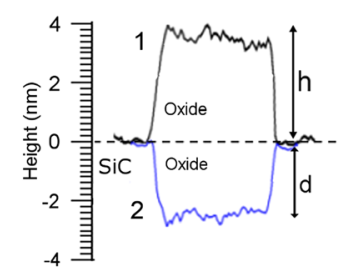

FIG. 3. (a) AFM 3D topography of the pattern prepared for the etching test, $251 \times 1 \mu \mathrm{m}$ oxide squares obtained by parallel lines written at $1 \mu \mathrm{m} \mathrm{s}{ }^{-1}$ and $10 \mathrm{~V}$ bias spaced $50 \mathrm{~nm}$. In (b), AFM topography of the same sample after being dipped into a mild hydrogen fluorine aqueous solution (5 wt. \%) for $30 \mathrm{~s}$. Height profile of the top left corner feature, before (black solid line) and after etching (blue solid line), is compared in (c) where the parameters $\mathrm{h}$ and $\mathrm{d}$ are also indicated.

combined with a very high electrical field below the tip. The reaction occurs at the $\mathrm{Si} / \mathrm{SiO}_{2}$ interface, the factor playing a key role is the transport of ionic oxidizing species across the growing silicon oxide layer, resulting in a self-limiting reaction. As the oxide layer grows, field strength $E_{\text {ext }}$ decreases, due to a thicker dielectric layer (in the parallel plate capacitor approximation $E_{\text {ext }}=V / h$ ). In addition to that, earlier studies by Avouris et al. ${ }^{22}$ identified the mechanical stress introduced into the growing oxide to explain apparent augmented activation energy in the oxidation process. Dagata et al. ${ }^{14,15}$ introduced the effect of space charges generated by the extremely high electric field conditions $\left(\mathrm{E}>10^{6} \mathrm{~V} / \mathrm{cm}\right)$ and fast initial growth. In this model, anionic species (i.e., $\mathrm{OH}^{-}$or other rate limiting species), initially formed from water dissociation, produce an accumulation of negative charges $\mathrm{Q}_{\max }$ at the tip/oxide interface that decays in time. At the same time, a certain percentage of oxanions, drifting through the growing oxide, is trapped producing additional space charge effects. The space charge region generated is responsible for the rapid reduction of the growth rate as the oxidation proceeds.

Considering what stated above, different kinetics laws were proposed. Dubois and Bubendorff, ${ }^{20}$ trying to account for space-charge limited growth, proposed a power-of-time law in the form $h(t, V)=h_{0}\left(t / t_{0}\right)^{1 /(\delta+1)}$ where $h, V$, and $t$ are the oxide thickness, the applied voltage, and the pulse time, respectively, $\mathrm{t}_{0}$ is a time constant depending on voltage and $\mathrm{h}_{0}$ and $\delta$ are fitting parameters. Dagata proposed a two paths explanation for the anodic oxidation: initially the direct oxidation process $\left(\mathrm{Si}+\mathrm{h}^{+}+2 \mathrm{OH}^{-} \rightarrow \mathrm{SiO}_{2}+2 \mathrm{H}^{+}\right)$is predominant, but over time a second indirect path of the oxidation occurs ${ }^{19}$ in which the charged defects are treated as reaction intermediates. Considering $\mathrm{SiC}$ instead of $\mathrm{Si}$, the direct anodic reaction becomes

$$
\mathrm{SiC}+8 \mathrm{~h}^{+}+4 \mathrm{OH}^{-} \rightarrow \mathrm{SiO}_{2}+4 \mathrm{H}^{+}+\mathrm{CO}_{2}
$$

where carbon atoms are completely eliminated in the form of carbon dioxide, as already mentioned carbon inclusion is also possible, leaving residuals with $\mathrm{SiO}_{\mathrm{x}} \mathrm{C}_{\mathrm{y}}$ stoichiometry (see Table I for proposed $\mathrm{x}$ and $\mathrm{y}$ values). The two models have been compared by Kinser et $a l .^{23}$ showing that the model based on two paths reaction better reproduces the data in case of FIO of $\mathrm{Si}$ in inert organic solvents. A comprehensive explanation of FIO kinetics is not yet available but the wide investigation made on Silicon FIO can be used for comparison. The electric field at the tip/oxide interface $\left(\mathrm{E}_{\mathrm{ext}}\right)$ generates the driving force necessary to overcome a certain potential barrier, inducing anions drift into the oxide. In a simple plane capacitor model, $\mathrm{E}_{\text {ext }}$ is approximated with $V / h$. Working at constant voltage, the reaction stops as soon as oxide thickness lowers the field below a (material dependent) critical value that, in case of $\mathrm{Si}$, corresponds to $h_{(\max )} \approx 6 \mathrm{~nm}$ and $\mathrm{E}_{\mathrm{ext}} \approx 10^{7} \mathrm{~V} \mathrm{~cm}^{-1},{ }^{22}$ close to the thermal diffusion limit. ${ }^{14}$ Surprisingly, in case of freshly etched $6 \mathrm{H} \mathrm{SiC}$, a critical thickness of $\approx 100 \mathrm{~nm}$ for $30 \mathrm{~s}$ pulses of $10 \mathrm{~V}$ is found, as shown in Figs. 1 and 2. Such a thickness is remarkably higher than what was previously reported on other materials (for $\mathrm{n}$ type $4 \mathrm{H} \mathrm{SiC}$ the highest value reported was $16 \mathrm{~nm})^{6}$ and opens up interesting possibilities for SPM three dimensional fabrication. Some considerations follow from this result: first, considering the expansion rate measured of 2.49 (Table I), the real critical oxide thickness $h_{\text {(max) }}=(h+d)$ is

TABLE I. Calculated (from values reported) and experimental expansion factors $\nu^{*}$ for different possible oxide composition. Reaction are not meant to describe the exact path but are reported for clarity.

\begin{tabular}{|c|c|c|c|c|c|c|}
\hline & u.m. & $\mathrm{Si}$ & $\mathrm{SiO}_{2}$ & $\mathrm{SiC}$ & $\mathrm{SiO}_{1.9} \mathrm{C}_{0.17}{ }^{\mathrm{a}}$ (C 5.5\%) low-C & $\mathrm{SiO}_{0.9} \mathrm{C}_{0.46}{ }^{\mathrm{a}}$ (C 19.7\%) high-C \\
\hline$\rho$ & $\mathrm{g} \mathrm{cm}^{-3}$ & 2.320 & $2.200^{\mathrm{b}}$ & 3.210 & 2.090 & 1.170 \\
\hline$M$ & $\mathrm{~g} \mathrm{~mol}^{-1}$ & 28.080 & 60.080 & 40.110 & 60.500 & 48.000 \\
\hline$m^{* \mathrm{c}}$ & $\mathrm{cm}^{3} \mathrm{~mol}^{-1}$ & 12.103 & 27.309 & 12.495 & 28.947 & 41.026 \\
\hline Reaction & & $\mathrm{Si} \rightarrow \mathrm{SiO}_{2}$ & & $\mathrm{SiC} \rightarrow \mathrm{SiO}_{2}$ & $\mathrm{SiC} \rightarrow \mathrm{SiOC}$ & $\mathrm{SiC} \rightarrow \mathrm{SiOC}$ \\
\hline$\nu^{*}$ calc. & & 2.26 & & 2.19 & 2.32 & 1.42 \\
\hline$h / d=\left(\nu^{*}-1\right)$ & & 1.26 & & 1.19 & 1.32 & 0.42 \\
\hline$\nu^{*}$ experim. & & $2.65^{\mathrm{d}}$ & & & \multicolumn{2}{|c|}{$2.49 \pm 0.31$} \\
\hline
\end{tabular}

${ }^{\mathrm{a}}$ From Ref. 9, SiOC films prepared by plasma-enhanced chemical vapor depositions on $\mathrm{Si}$.

${ }^{\mathrm{b}}$ Density of thermal-wet grown film.

${ }^{c} m^{*}$ is the molar volume $\mathrm{M} / \rho ; \nu^{*}=m^{*}(O x.) / m^{*}(\operatorname{Sub}$. $)$.

${ }^{\mathrm{d}}$ From Ref. 15. 
$167 \mathrm{~nm}$. This brings to $\mathrm{E}_{\mathrm{ext}} \approx 6 \times 10^{5} \mathrm{~V} \mathrm{~cm}^{-1}$ that is considerably lower $\left(10^{2}\right.$ times) with respect to the electric field threshold, indicating a drop in the activation energy for ion migration process. Second, Dagata states that during the initial stages of FIO the initial charge density $\mathrm{Q}_{\max } / \mathrm{A}$ (Ref. 24) (with $\mathrm{Q}_{\max }$ as ion charge at the tip-substrate junction and $\mathrm{A}$ as the effective contact area) is ruling the following reaction rates. In both cases ( $\mathrm{SiC}$ or $\mathrm{Si} \mathrm{FIO),} \mathrm{water} \mathrm{will} \mathrm{readily} \mathrm{ion-}$ izes and the condensation condition are identical. So, if initial anions concentration is the same, such a difference in the limiting thickness must depend on an improved diffusion mechanism. It is recognized that water molecule diffusion in oxides depends strongly on density and specifically, the activation energy $E_{a}$ decreases linearly with an increase in ionic porosity $\mathrm{Z}=1-m_{I}^{*} / m^{*}, 25$ where $m^{*}$ is the bulk molar volume $\mathrm{M} / \rho$ and $m_{I}^{*}$ is the molar volume of the atoms composing the cell $\left(m_{I}^{*}=\sum\left[4 \pi r_{i} / 3\right] N\right.$, where $r_{i}$ are the ionic radii and $N$ is the Avogadro's number). For thermal oxide $\mathrm{Z} \approx 0.50$ that brings to a value of $\mathrm{E}_{\mathrm{a}}$ for molecular water diffusion in silica glass of approximately $71 \mathrm{~kJ} \mathrm{~mol}^{-1} \cdot{ }^{25,26}$ In the case of $\mathrm{SiC}$ oxide (FIO $\mathrm{SiO}_{2}$ ), $m_{I}^{*}$ is unchanged and $m^{*}=M_{\mathrm{SiO}_{2}} / \rho$, where the value of $\rho$ is the one estimated previously $\left(1.93 \mathrm{~g} \mathrm{~cm}^{-3}\right)$. This leads to a value of $Z^{\prime} \approx 0.56$ for the FIO oxide. As mentioned above, Ref. 24 gives an empirical correlation between $\mathrm{E}_{\mathrm{a}}$ and $\mathrm{Z}\left(\mathrm{E}_{\mathrm{a}}=494-821 * \mathrm{ZkJ} \mathrm{mol}^{-1}\right)$. Using that correlation, we can conclude that the activation energy for water diffusion in FIO oxide grown on $\mathrm{SiC}$ is approximately $34 \mathrm{~kJ} \mathrm{~mol}^{-1}$, indicating again a facilitated ion migration within the oxide grown on $\mathrm{SiC}$.

The series of dots fabricated by contact mode FIO (Figs. 1 and 4) with different pulse times have been used to collect a set of experimental data from which extract some kinetics considerations. We varied the pulse time and the voltage applied operating in a humidity range (20\% to $45 \%$ ) in which water capillary condensation is guaranteed but lateral diffusion is minimized. ${ }^{24}$ The dependence of the oxide height on RH at constant voltage and reaction time (10 V; $5 \mathrm{~s}$ and $8 \mathrm{~V} ; 10 \mathrm{~s}$, respectively) is shown in Fig. 4(a). Rather than varying humidity, we concentrated on obtaining arrays of dots at fixed RH, changing voltage and pulse times, the two parameters that are more widely reported in literature. The oxide dots shown in Figs. 4(a) and 4(b) were obtained during a single pass at $\mathrm{RH}=25 \%$, while the series of dots used to model the kinetics were obtained at the same humidity used in Ref. 6; $\mathrm{RH}=40 \%$. Data are in agreement with the previous SPL oxidation literature, with oxide height growth rates $(d h / d t)$ similar to Si FIO (Fig. 4(d)), and a self limiting behavior is well reproduced by the so called modified Avouris rate equation ${ }^{18}$ in which

$$
d h / d t=R \exp \left(-E_{0} h / V\right),
$$

where $\mathrm{V}$ is the applied voltage, $\mathrm{h}$ is the oxide dot thickness, $\mathrm{R}$ and $\mathrm{E}_{0}$ are fitting parameters. The integration of Eq. (1) leads to a direct $\log$ of time dependence in the form

$$
h(t, V)=V / E_{0} \ln \left[R E_{0} t / V+1\right] .
$$

In Figs. 4(b) and 4(c), we show the topography (c) and dots height vs. pulse time (b) obtained with a single pass modulating both voltage and pulse time. Equation (2) well fits
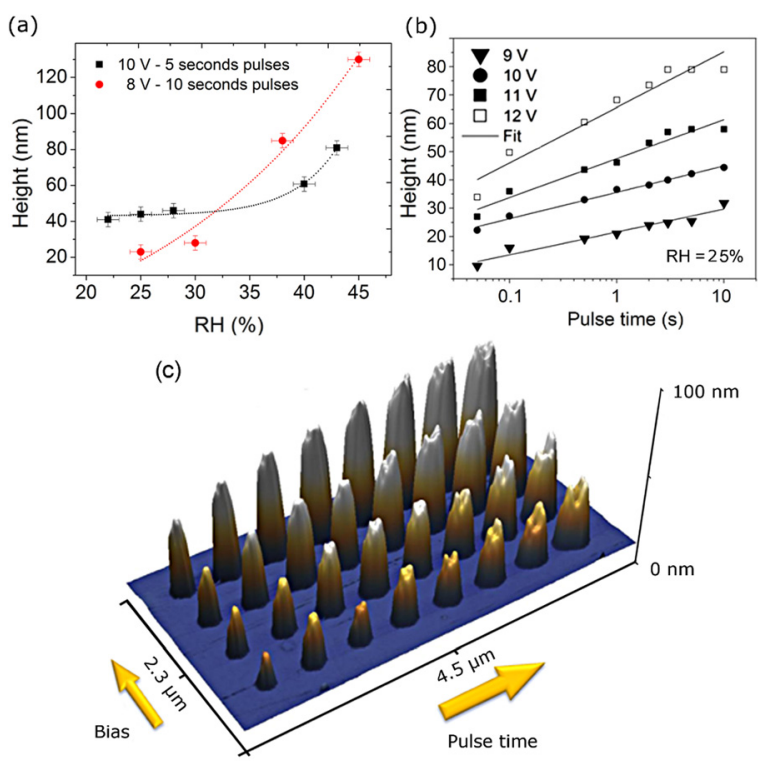

(d)

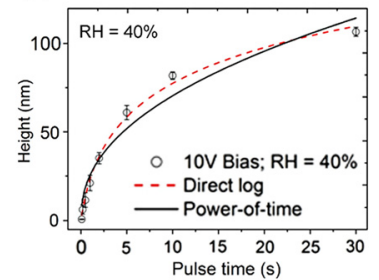

(e)

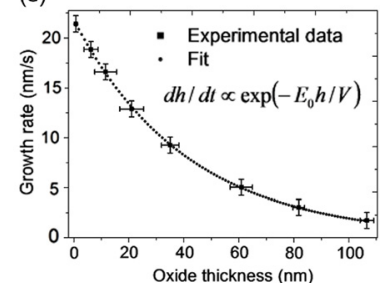

FIG. 4. Kinetics study of tip induced oxidation on $6 \mathrm{H}-\mathrm{SiC}$. (a) The dependence of the oxide height on RH at constant voltage and reaction time (10 V; $5 \mathrm{~s}$ and $8 \mathrm{~V} ; 10 \mathrm{~s}$, respectively), the dotted lines are fit with an exponential decay given as an eye guide. (b) Plot of dots eight versus pulse duration (log scale) for a series of features grown by single pulse oxidation, the data are taken from the AFM scan shown in (c). Voltage pulse time varies from left to right ranging from 0.05 to $10 \mathrm{~s}$. Voltages applied on each row were 9,10 , 11 , and 12 , respectively. The oxide height increases as the logarithm of the pulse time and, for a constant oxidation time, increases linearly with the applied voltage. (d) The average eight of the dots arrays shown in Fig. 1 is plotted versus the pulse duration (linear scale). The dots shown in (d) were produced at a relative humidity of $40 \%$. The solid black line represents the fit with a power-of-time law while the dashed line represents the fit with a modified Avouris rate equation (direct log-of-time). (e) Oxidation growth rate versus the oxide thickness showing how the rate falls off exponentially with oxide thickness.

experimental data for different voltage applied (Fig. 4(b)). In order to expand the exposure time scale and to ensure the reproducibility of the measures, we produced 10 identical arrays of single dots. The result is shown in Figure 1(a) (for better visualization 5 arrays are shown) while dots height vs. pulse time values are plotted in Fig. 4(d). Each line is obtained by the same pulse variation, eight pulses ranging from 0.1 to $30 \mathrm{~s}$. The voltage applied was $10 \mathrm{~V}$ and the relative humidity kept at $40 \%$. Also in this case, direct log-oftime law better captures the data (correlation coefficient $\mathrm{R}=0.997$ with $\mathrm{E}_{0}=0.32 \mathrm{~V} / \mathrm{nm}$ and $\mathrm{R}=34.4 \mathrm{~nm}$ ) while the power-of-time $\operatorname{law}^{20,23}$ does not fit satisfactorily. In Fig. 4(e), the oxidation rate versus oxide thickness is plotted, showing the exponential fall off with $h$ of the rate.

Last, space charge effects decrease when oxide thickness exceeds few nanometers. In order to better understand the role of space charge during $\mathrm{SiC}$ oxidation, we performed the measurement of the residual space charge, as proposed by Chiesa and Garcia. ${ }^{27}$ The space charge density can be 
measured by mean of KPFM, since the surface potential is sensitive to the presence of trapped charges. By assuming a uniform charge density and solving the Poisson equation, as in Ref. 26, the relation between the measured surface potential $\left(\mathrm{V}_{\mathrm{s}}\right)$ and the charge density $\rho$ can be deduced

$$
\rho=\frac{2 \varepsilon \varepsilon_{0}}{h^{2}} \Delta \mathrm{V}_{\mathrm{s}}
$$

where $h$ is the total thickness of the oxide, $\varepsilon$ is the relative dielectric constant of the oxide, $\varepsilon_{0}$ is the vacuum dielectric constant, and $\Delta \mathrm{V}_{\mathrm{s}}$ is the difference in surface potential between the grounded substrate and the oxide. We performed KPFM measurements in dry $\mathrm{N}_{2}$ environment $(\mathrm{RH}<4 \%)$ on both kinds of oxides, few minutes after the features were produced. As shown in Fig. 5(a), for oxide pattern fabricated on a p-type Si (100) substrate the sign of the trapped charges measured is negative and the residual charge density is found to be $\sim 10^{17} \mathrm{~cm}^{-3}$, in agreement with what was reported by Chiesa and Garcia. Interestingly the value of the local surface potential (that has the same sign of the charge) for oxide pattern fabricated on an n-type $6 \mathrm{H}-\mathrm{SiC}(0001)$ substrate is positive with respect to the grounded $\mathrm{SiC}$ (Figure 5(b)). For $\mathrm{h}=6.1 \mathrm{~nm}$ and $\varepsilon=3.9$ (Ref. 27), the calculated space charge density is $4.6 \times 10^{17} \mathrm{~cm}^{-3}$. Excluding the screening effect of adsorbed water $(\mathrm{RH}<4 \%)$, this effect is explained by positive charge accumulation in the oxide features, which would enhance the oxidation process rather than limiting it. (a)
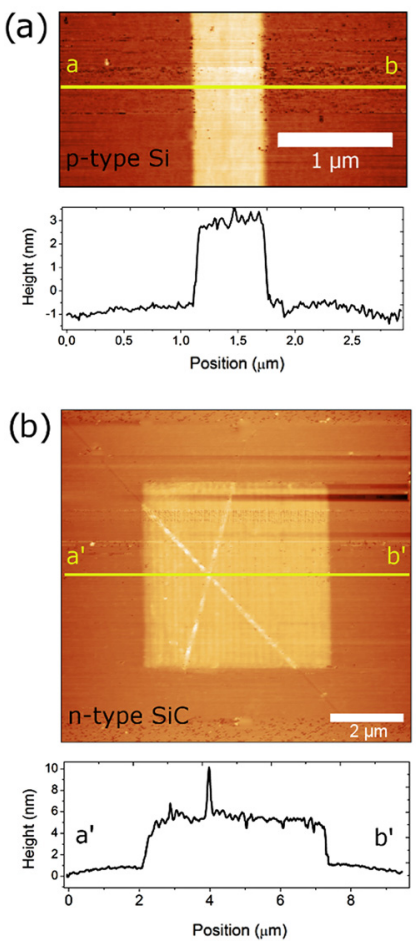
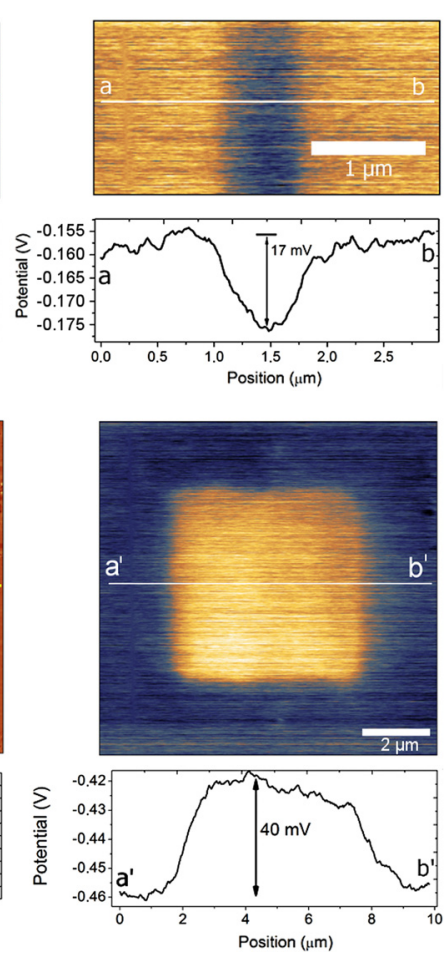

FIG. 5. (a) AFM topography of an oxide pattern fabricated on a p-type $\mathrm{Si}(100)$ (left) and correspondent surface potential image (right); each image is accompanied by the profile across the sections a and b. (b) AFM topography of an oxide pattern fabricated on a n-type $6 \mathrm{H}-\mathrm{SiC}(0001)$ (left) and correspondent surface potential image (right); each image is accompanied by the profile across the section $\mathrm{a}^{\prime}-\mathrm{b}^{\prime}$. For $\mathrm{Si}(\mathrm{a})$, the $\Delta \mathrm{V}_{\mathrm{s}}$ between the grounded substrate and the oxide is negative $(-17 \mathrm{mV})$ while for $\mathrm{SiC}(\mathrm{b})$ the difference is positive $(40 \mathrm{mV})$.
In summary, we explored the potential of scanning probe oxidation on $6 \mathrm{H}-\mathrm{SiC}$ substrates demonstrating that very high oxide features, characterized by very high aspect ratio, can be fabricated. This improvement in the $3 \mathrm{D}$ characteristic of the oxide can be exploited to fabricate masks by means of simple dry etching, opening up new possibilities for soft lithography and other applications. Oxide growth kinetics on $\mathrm{SiC}$ has been described in terms of theoretical and empirical models introduced for $\mathrm{Si}$, finding that $\mathrm{SiC}$ FIO process in air is well described by the so called modified Avouris rate equation in which the growth rate decays exponentially. For longer oxidation times, the kinetics models accounting for space charge effect fail in reproducing the experimental data, while the empirical Avouris rate equation well fits the data collected. This augmented oxide thickness on $\mathrm{SiC}$ can be semi-quantitatively explained in terms of oxide density and trapped charges during FIO. These two parameters have been indirectly measured by means of etching tests and KPFM, concluding that carbon content in $\mathrm{SiO}_{2}$ on $\mathrm{SiC}$ (not present in $\mathrm{SiO}_{2} / \mathrm{Si}$ case) causes, on one side, a drop in the activation energy for oxyanions diffusion within the oxide layer while, on the other hand, a considerable decrease of the effect of space charge. Those conclusions lead to interesting possibilities for further investigation in the field of SPL, such as the characterization of the oxide produced by SPL (either on $\mathrm{SiC}, \mathrm{Si}$, or other materials) in terms of density and water diffusion behavior.

This work was entirely supported by the Italian Institute of Technology (IIT); a special appreciation for the support coming from Lorenzo Ferrara and Eliana Rondanina.

${ }^{1}$ X. N. Xie, H. J. Chung, C. H. Sow, and A. T. S. Wee, Mater. Sci. Eng. R 54, 1 (2006).

${ }^{2}$ A. A. Tseng, L. Pellegrino, and J.-I. Shirakashi, in Encyclopedia of Nanoscience and Nanotechnology, 2nd ed., edited by H. S. Nalwa (American Scientific Publishers, Valencia, 2012), pp. 171-207.

${ }^{3}$ R. Garcia, R. V. Martinez, and J. Martinez, Chem. Soc. Rev. 35, 29 (2006).

${ }^{4}$ M. Lorenzoni, A. Giugni, and B. Torre, Nanoscale Res. Lett. 8, 75 (2013).

${ }^{5}$ X. N. Xie, H. J. Chung, H. Xu, X. Xu, C. H. Sow, and A. T. S. Wee, J. Am. Chem. Soc. 126, 7665 (2004).

${ }^{6}$ Y. Jo, S. Seo, W. Bahng, and S. Kim, Appl. Phys. Lett. 96, 082105 (2010).

${ }^{7}$ J.-J. Ahn, Y.-D. Jo, S.-C. Kim, J.-H. Lee, and S.-M. Koo, Nanoscale Res. Lett. 6, 235 (2011).

${ }^{8}$ S. Dhar, O. Seitz, M. D. Halls, S. Choi, Y. J. Chabal, and L. C. Feldman, J. Am. Chem. Soc. 131, 16808 (2009).

${ }^{9}$ See supplementary material at http://dx.doi.org/10.1063/1.4825265 for materials, experimental details, AFM topography of $\mathrm{SiC} 6 \mathrm{H}-0001$ (Si) surface CMP polished, conductive AFM measurements and additional examples of patterns obtained.

${ }^{10}$ W. N. Hansen and G. J. Hansen, Surf. Sci. 481, 172 (2001).

${ }^{11}$ M. Tello and R. García, Appl. Phys. Lett. 79, 424 (2001).

${ }^{12}$ P. Yih, V. Saxena, and A. Steckl, Physica Status Solidi B 202, 605 (1997).

${ }^{13}$ C. Canale, B. Torre, D. Ricci, and P. C. Braga, Methods Mol. Biol. 736, 31 (2011).

${ }^{14}$ J. A. Dagata, T. Inoue, J. Itoh, K. Matsumoto, and H. Yokoyama, J. Appl. Phys. 84, 6891 (1998).

${ }^{15}$ K. Morimoto, F. Pérez-Murano, and J. Dagata, Appl. Surf. Sci. 158, 205 (2000).

${ }^{16}$ H. J. Kim, Q. Shao, and Y.-H. Kim, Surf. Coat. Technol. 171, 39 (2002).

${ }^{17}$ P. Avouris, T. Hertel, and R. Martel, Appl. Phys. Lett. 71, 285 (1997).

${ }^{18}$ E. S. Snow, G. G. Jernigan, and P. M. Campbell, Appl. Phys. Lett. 76, $1782(2000)$

${ }^{19}$ J. A. Dagata, F. Perez-Murano, G. Abadal, K. Morimoto, T. Inoue, J. Itoh, and H. Yokoyama, Appl. Phys. Lett. 76, 2710 (2000).

${ }^{20}$ E. Dubois and J.-L. Bubendorff, J. Appl. Phys. 87, 8148 (2000). 
${ }^{21}$ F. P. Fehlner and N. F. Mott, Oxid. Met. 2, 59 (1970).

${ }^{22}$ P. Avouris, R. Martel, T. Hertel, and R. Sandstrom, Appl. Phys. A: Mater. Sci. Process. 66, S659 (1998).

${ }^{23}$ C. R. Kinser, M. J. Schmitz, and M. C. Hersam, Adv. Mater. 18, 1377 (2006).
${ }^{24}$ J. A. Dagata, F. Perez-Murano, C. Martin, H. Kuramochi, and H. Yokoyama, J. Appl. Phys. 96, 2386 (2004).

${ }^{25}$ R. H. Doremus, J. Mater. Res. 14, 3754 (1999).

${ }^{26}$ M. Calleja and R. Garcia, Appl. Phys. Lett. 76, 3427 (2000).

${ }^{27}$ M. Chiesa and R. Garcia, Appl. Phys. Lett. 96, 263112 (2010). 